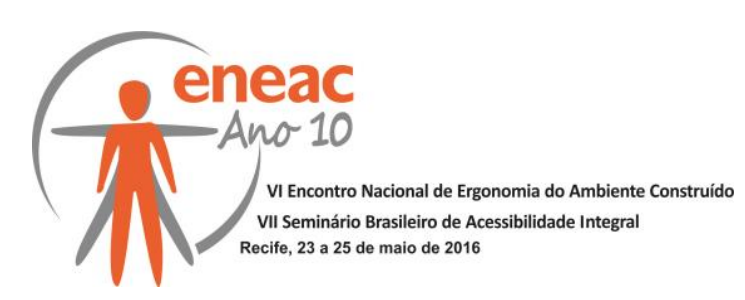

\title{
ANÁLISE ACÚSTICA DO AUDITÓRIO PAULO BONAVIDES NAS FACULDADES INTEGRADAS DE PATOS/PB
}

\author{
GOMES, Emmily Gersica Santos (1); \\ LUCENA, Pedro Gomes de (2); \\ CUNHA, Marcella Viana Portela de Oliveira (3); \\ ALVES, Anderson Ramon Lopes (4);
}

(1) Faculdades Integradas de Patos, Mestre em Arquitetura e Urbanismo

e-mail: emmilygersica@hotmail.com

(2) Faculdades Integradas de Patos, Graduando em Arquitetura e Urbanismo

e-mail: pedroglucena@gmail.com

(3) Faculdades Integradas de Patos, Mestre em Arquitetura e Urbanismo

e-mail: marcellaportela@hotmail.com

(4) Faculdades Integradas de Patos, Graduando em Arquitetura e Urbanismo

e-mail: and ramon@hotmail.com

\begin{abstract}
RESUMO
Este artigo trata da análise das condições acústicas do auditório Paulo Bonavides nas Faculdades Integradas de Patos, onde, após analises, constatou-se que o referido auditório se encontra com condições inadequadas de audibilidade, provocado por um comportamento inadequado do som, devido a presença de materiais com capacidade absortiva insatisfatória. Foi realizada proposta de correção acústica a partir de simulações que envolveram a mudança de materiais presentes no local, de modo a favorecer uma aproximação ao tempo ótimo de reverberação calculado, aliando intervenções adequadas para o uso específico com resultados satisfatórios.
\end{abstract}

Palavras chave: Acústica, Reverberação, Auditório.

\begin{abstract}
This article deals with the analysis of the acoustics of Paul Bonavides auditorium on the Integrated Faculties of Patos where the survey found that that audience is with inadequate conditions of sound, caused by a misbehaving sound because the site does not have enough absorptive materials. Acoustic correction proposed was based simulations involving the change of materials present on site, in order to favor an approach to optimal reverberation time calculated.
\end{abstract}

Keywords: Acoustics, Reverberation, Auditorium. 


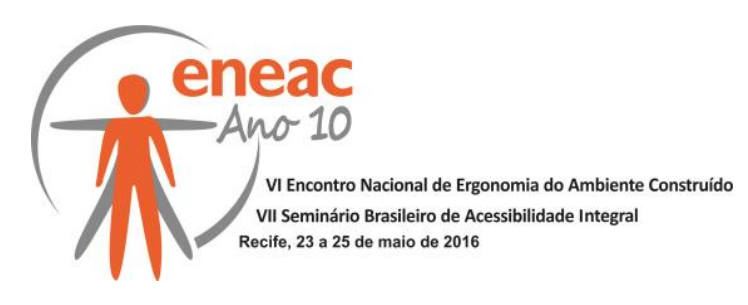

\section{INTRODUÇÃO}

Ao longo dos últimos anos tem sido recorrente a necessidade de pesquisar e levar em consideração os conceitos e a contribuição técnica da acústica arquitetônica para o espaço edificado. O Controle das condicionantes acústicas a partir da análise e interpretação dos níveis de ruído, absorção sonora, da reverberação e do isolamento sonoro possibilitam o desenvolvimento de ambientes que garantam a qualidade do som conforme o uso e tipologia necessária.

Quando nos referimos à qualidade do som de um espaço versamos estes conceitos a uma série de condicionantes que refletem diretamente nas experiências auditivas do indivíduo, principalmente ao se fazer uso de ambientes sintetizados acusticamente.

Nesta perspectiva, constatou-se que o objeto de estudo desta pesquisa, o auditório Paulo Bonavides das Faculdades Integradas de Patos, na cidade de Patos/PB, possui a necessidade de verificação da situação atual do ambiente, tanto nos aspectos físicos, quanto acústicos. Foi possível observar que durante o processo de projeto foram adotadas algumas medidas, que visassem o conforto acústico, tais como: piso e forro escalonados, carpetes em partes do piso, entre outros. A partir do levantamento arquitetônico e do conhecimento da área em questão, foi possível estudar o comportamento sonoro no recinto, para assim, propor uma intervenção que vise a melhoria da acústica no ambiente.

Por se tratar de estabelecimento de ensino, a função principal do espaço é a realização de palestras, cursos, seminários e outras atividades que dão suporte a vida acadêmica, portanto, a acessibilidade sonora é essencial para que as pessoas possam se comunicar de forma adequada, tornando-se possível a inteligibilidade das comunicações. De tal modo, para se obter a qualidade da função desejada deve-se detalhar os materiais, objetos e elementos, além dos fenômenos que envolvem os caminhos do som dentro do ambiente estudado, evidenciando o nível acústico e as necessidades de modificação do espaço, e assim propor intervenções para a correção acústica do local.

Com base no exposto, o estudo ganha importância ao colaborar com a disseminação do conhecimento a respeito do conforto acústico, levando em consideração seus princípios e finalidades, contribuindo para nortear os projetos que buscam melhorias de promoção da qualidade do ambiente edificado, ratificando a necessidade de se propor condições favoráveis para a realização de atividades nos diversos espaços da sociedade, além de evidenciar a importância da elaboração de projetos comprometidos com o conforto acústico, sendo necessário o conhecimento e embasamento técnico para que tal proposição se torne realidade.

\section{OBJETO DE ESTUDO}

O auditório Paulo Bonavides fica localizado no primeiro andar do bloco B da unidade II das Faculdades Integradas de Patos, em Patos-PB, ocupando uma área de $107.29 \mathrm{~m}^{2}$, com dimensões de $7.53 \mathrm{~m}$ de largura por $14.34 \mathrm{~m}$ de comprimento e pés-direitos variáveis com uma altura média de $3,00 \mathrm{~m}$ (figura 1). O espaço é destinado a realização de atividades educativas da Instituição, onde a principal função desenvolvida no seu interior, é a palavra falada. Possui no seu entorno, salas de aula, sendo necessário o condicionamento acústico, para a interrupção de fluxos de ruídos. 


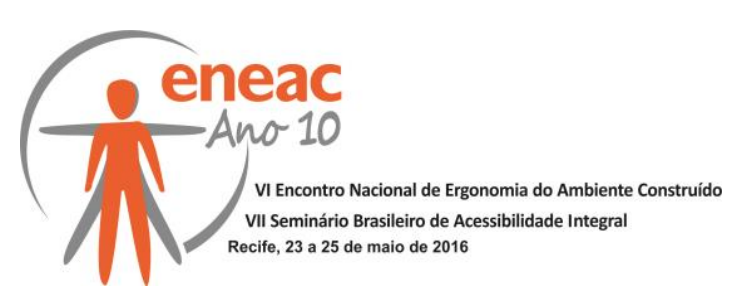

Figura 1 - Planta baixa do auditório Paulo Bonavides.

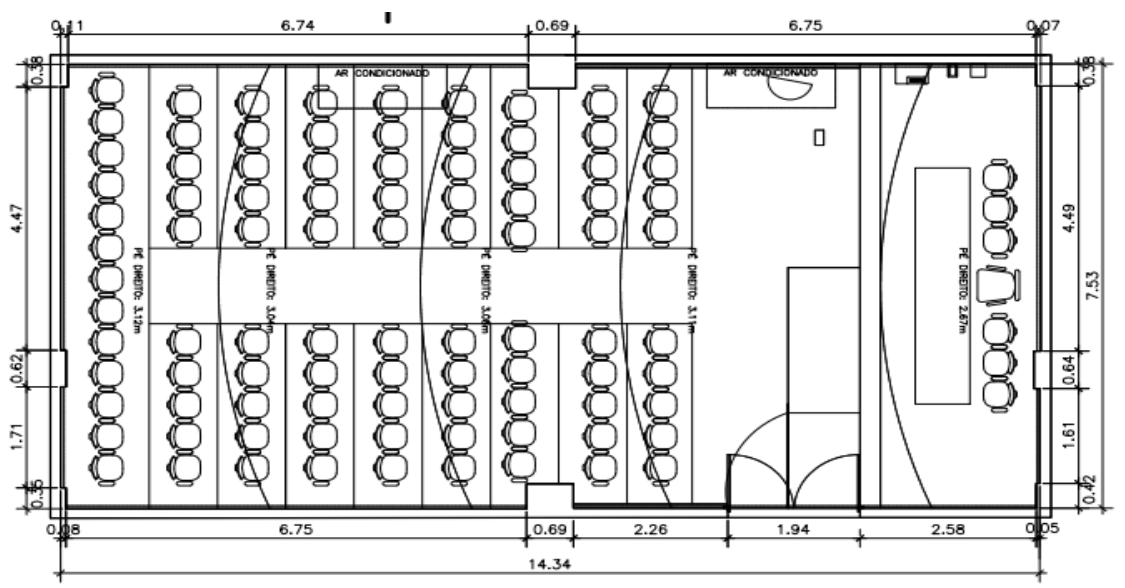

Fonte - Autores, 2015.

O acesso a sala é feito por meio de uma porta de madeira compensada envernizada com dimensões de $1.90 \mathrm{~m} \times 2.10 \mathrm{~m}$. As paredes do ambiente são constituídas de tijolo cerâmico, reboco e tinta acrílica, além disso, há a presença de uma moldura de madeira de aproximadamente $15 \mathrm{~cm}$ disposta horizontalmente no centro das paredes, dividindo-as em dois seguimentos, um revestido com tinta acrílica e outro com revestimento texturizado.

O piso apresenta diferenças de níveis, com um segmento escalonado na parte dos assentos e o palco com elevação de $30 \mathrm{~cm}$. O revestimento do piso na parte da rampa central que dá acesso aos assentos é de carpete, já a parte escalonada que compreende a plateia e a parte do palco, é em cerâmica. O material do teto é gesso, e possui uma forma escalonada (figura 2), favorecendo uma melhor distribuição das ondas sonoras em todo o espaço (figura 3).

Figura 2 - Visão do auditório.

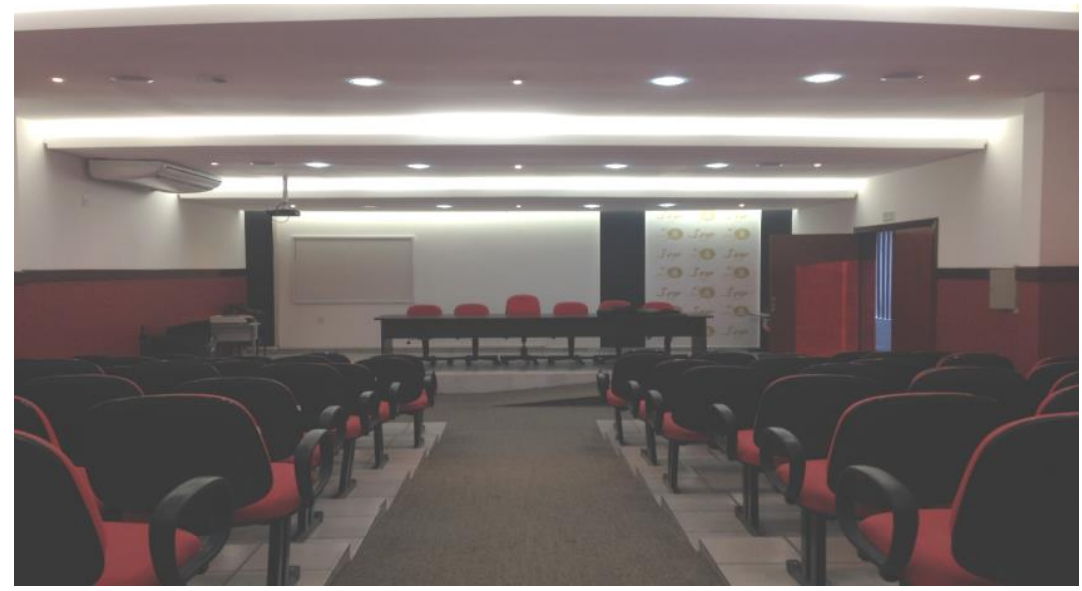

Fonte - Autores, 2015. 


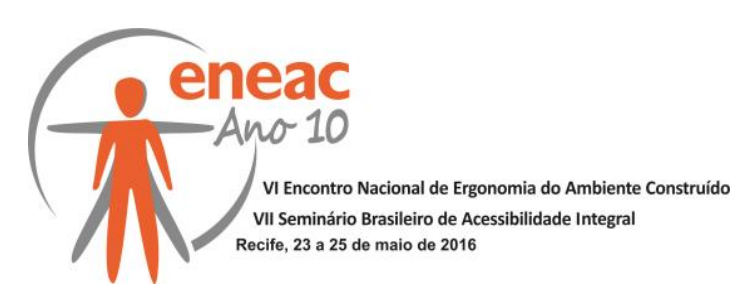

Figura 3 - Esquema de distribuição das ondas sonoras em função da geometria do teto.

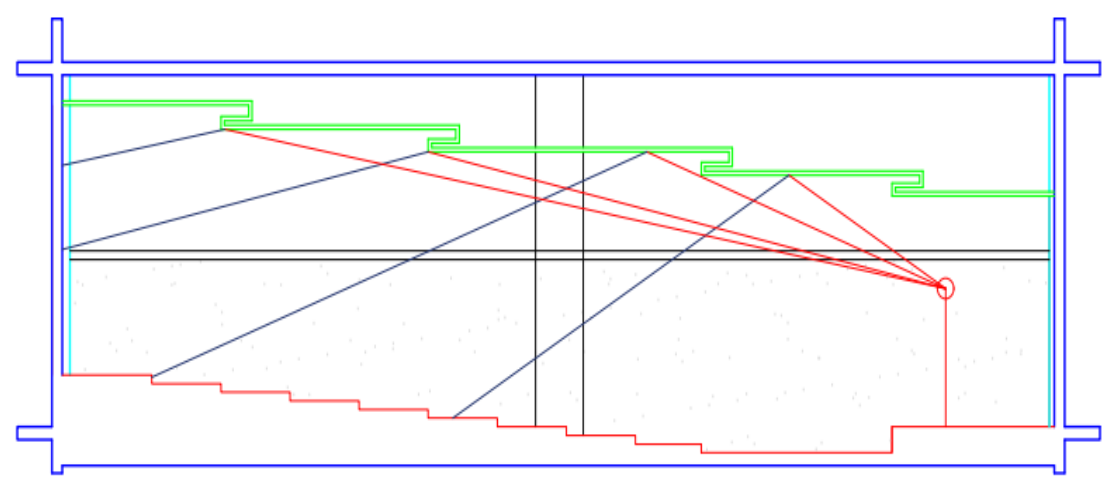

Fonte - Autores, 2015.

Observa-se na tabela 1 a seguir, o levantamento das superfícies aparentes no interior do auditório. É importante que seja levantado o quantitativo de cada material presente no local, para posterior cálculo da influência dos mesmos nos fenômenos acústicos no interior do recinto.

Tabela 1 - Características do Ambiente Existente com capacidade para 98 lugares, e com volume aproximado de $323,94 \mathrm{~m}^{3}$.

\begin{tabular}{|c|c|}
\hline Material & Área \\
\hline Forro de gesso & $105,2 \mathrm{~m}^{2}$ \\
\hline Piso cerâmico: & $77.72 \mathrm{~m}^{2}$ \\
\hline Piso mármore: & $1,8 \mathrm{~m}^{2}$ \\
\hline Piso e rampas em carpete simples: & $29.02 \mathrm{~m}^{2}$ \\
\hline Porta de acesso em madeira compensada pintada: & $3.99 \mathrm{~m}^{2}$ \\
\hline Parede lateral direita em alvenaria pintada: & $21.7 \mathrm{~m}^{2}$ \\
\hline Parede lateral direita com textura: & $25.88 \mathrm{~m}^{2}$ \\
\hline Detalhe em madeira parede lateral direita: & $1,50 \mathrm{~m}^{2}$ \\
\hline Parede lateral esquerda em alvenaria pintada & $21.7 \mathrm{~m}^{2}$ \\
\hline Parede lateral esquerda com textura & $25.88 \mathrm{~m}^{2}$ \\
\hline Detalhe em madeira parede lateral direita: & $1,31 \mathrm{~m}^{2}$ \\
\hline Parede fundo em alvenaria pintada: & $15.78 \mathrm{~m}^{2}$ \\
\hline Parede fundo em alvenaria com textura: & $9,45 \mathrm{~m}^{2}$ \\
\hline Detalhe em madeira parede de fundo: & $0,78 \mathrm{~m}^{2}$ \\
\hline
\end{tabular}




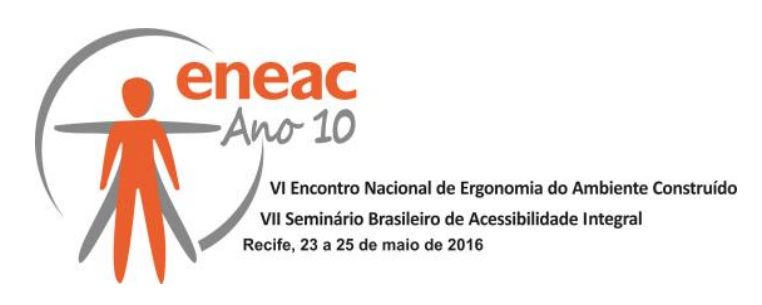

Parede frontal (palco) em alvenaria pintada:

$23.01 \mathrm{~m}^{2}$

Na tabela 2 a seguir, temos o quantitativo de mobiliário presente no auditório.

Tabela 2 - Material, quantidade e área do mobiliário presente no auditório.

\begin{tabular}{|c|c|c|}
\hline Item & Quantidade & Área \\
\hline Poltrona estofada (poliéster) & 98 & $45,0 \mathrm{~m}^{2}$ (total) \\
\hline Mesa (madeira) & 01 & $3,2 \mathrm{~m}^{2}$ \\
\hline Auto falante embutido (plástico) & 10 & $0,031 \mathrm{~m}^{2}$ (cada) \\
\hline Spot lamp. fluorescente (vidro) & 20 & $0,0176 \mathrm{~m}^{2}$ (cada) \\
\hline Spot lamp. halogênica (vidro) & 15 & $0,078 \mathrm{~m}^{2}$ (cada) \\
\hline Central de ar (plástico) & 02 & $1,42 \mathrm{~m}^{2}$ (cada) \\
\hline
\end{tabular}

\section{REFERENCIAL TEÓRICO}

A acústica é o ramo da Física que estuda e analisa as ondas sonoras, a sua propagação em meios elásticos, tanto fluidos como sólidos, e as suas inter-relações com o ser humano. Estas distinguem-se consoante, possuam ou não carácter de agradabilidade e significado, correspondendo a som ou a ruído, respetivamente. (PINTO, 2012)

Segundo De Marco (1982 apud SILVA ,2012), o projeto de auditórios é um dos mais complexos dentro da acústica arquitetônica e um dos principais problemas a serem enfrentados é a redução do ruído a níveis muito baixos, tanto os ruídos externos quanto internos. O projeto de auditórios exige um cálculo preciso das características absorventes e refletoras dos materiais de acabamento para um uso específico. Em uma intervenção de um auditório existente a tarefa torna-se ainda mais árdua.

De acordo com o artigo 2ํำ do Regulamento dos Requisitos Acústicos dos Edifícios (RRAE), o tempo de reverberação, em termos energéticos é o intervalo de tempo necessário para que a energia volumétrica do campo sonoro de um recinto fechado se reduza a um milionésimo do seu valor inicial $(60 \mathrm{~dB})$, depois da fonte ter parado. Ou seja, é o tempo de permanência do som no ambiente, e esse fenômeno está diretamente ligado à capacidade que os materiais presentes no recinto tem de refletir o sinal sonoro.

Conforme Bastos (2012), o tempo de reverberação está intimamente ligado à caracterização da acústica do ambiente pois seu valor pode ser favorável ou não para a qualidade da palavra, visto que esse fenômeno pode aumentar o nível sonoro ou mascarar o som direto, classificando as salas como "vivas" quando têm tempos de reverberação mais significativos e "mortas" quando quase não existe reverberação. Essa é inversamente proporcional a capacidade de absorção sonora dos materiais, pois quanto maior for essa capacidade no ambiente, menor o tempo de reverberação. A absorção corresponde ao quociente entre a quantidade de energia sonora absorvida por um certo material e a quantidade de energia que sobre ele incide e esse quociente varia de 0 a 1. 


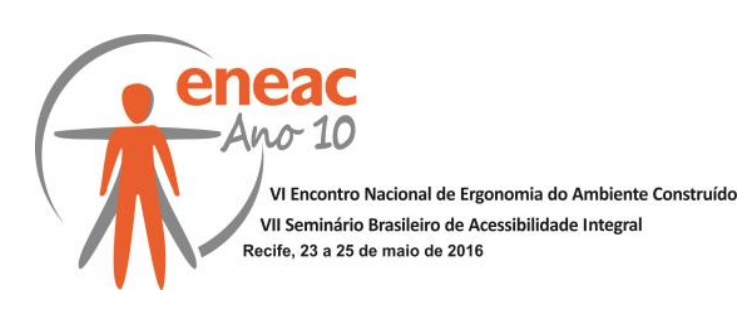

De acordo com a NBR 12179 (ABNT, 1992), o cálculo dos coeficientes de absorção e o tempo de reverberação se dá a partir da equação de Sabine, conforme equação a seguir:

$$
\mathrm{TR}=0,161 . \mathrm{V} /(\text { Snan+Snan+...) }
$$

Onde:

$\mathrm{TR}=$ tempo de reverberação do recinto,

$\mathrm{V}=$ volume do recinto,

Snan = área das superfícies e seu respectivo coeficiente de absorção.

A absorção e reverberação estão intimamente relacionadas ao tipo de frequência do som, portanto, é imprescindível que sejam isoladas seis frequências distintas, analisando os comportamentos desses fenômenos nas mesmas, para assim, ser possível observar a situação atual do comportamento do som em diferentes momentos e a partir disto, propor a correção acústica ao ambiente.

O tempo ótimo de reverberação é determinado em função do volume do ambiente e seu uso, bem como pela frequência dos sons em relação à atividade desempenhada no espaço. A seguir pode-se ver na tabela 3, os tempos de reverberação em função do volume/uso e na tabela 4, os tempos de reverberação em função da frequência/uso, aconselháveis conforme previsto por Costa (2003).

Tabela 3 - Tempo de reverberação (s) aconselhável em função do volume $\left(\mathrm{m}^{3}\right)$.

\begin{tabular}{|c|c|c|c|}
\hline TIPO DE SOM & V: $\mathbf{1 0 0 0}$ & V: $\mathbf{2 5 0 0}$ & V: $\mathbf{5 0 0 0}$ \\
\hline Música (reproduzida) & 0,95 & 1,10 & 1,25 \\
\hline Música (direta) & 1,25 & 1,45 & 1,65 \\
\hline Linguagem & 0,83 & 0,93 & 1,02 \\
\hline
\end{tabular}

Tabela 4 - Tempo de reverberação (s) aconselhável em função da frequência ( $\mathrm{Hz})$.

\begin{tabular}{|c|c|c|c|}
\hline $\begin{array}{c}\text { TIPO DE } \\
\text { SOM }\end{array}$ & $\mathbf{F :} \mathbf{1 2 5 H z}$ & $\mathbf{F}: \mathbf{5 0 0 H z}$ & $\mathbf{F}: \mathbf{2 0 0 0 H z}$ \\
\hline Música & 2,0 & 1,0 & 1,1 \\
\hline Linguagem & 1,3 & 1,0 & 1,1 \\
\hline
\end{tabular}

De acordo com o Regulamento dos Requisitos Acústicos dos Edifícios (RRAE), em recintos cuja principal valência corresponda a atividades assentes na oratória, nomeadamente auditórios, o tempo de reverberação, $\mathrm{T}$, correspondente à média aritmética dos valores obtidos para as bandas de oitava, centradas nas frequências de $500 \mathrm{~Hz}, 1000 \mathrm{~Hz}$ e $2000 \mathrm{~Hz}$. Porém, como no auditório em questão a principal atividade centra na oratória, faz-se necessário o uso das frequências da fala: 125, 500 e $2000 \mathrm{~Hz}$, onde, o tempo de 


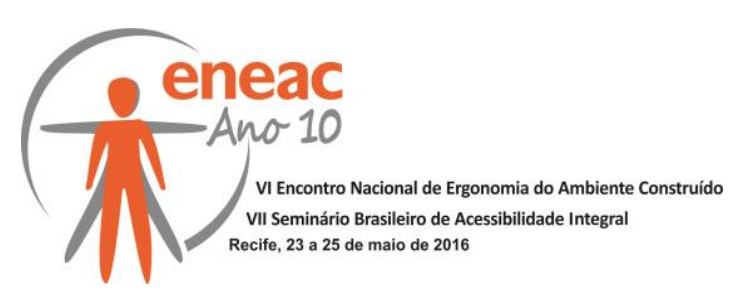

reverberação da situação em questão deverá corresponder, seguindo a lógica do RRAE, à média aritmética das frequências supracitadas.

\section{METODOLOGIA}

Para a realização da pesquisa, dividiu-se o processo em três partes: A primeira, constituiu o levantamento de dados, onde foram colhidas informações a respeito das técnicas e dos materiais de construção, bem como o mobiliário presente no ambiente. Para isso, foram realizadas visitas in loco para obtenção de registros fotográficos utilizando máquina fotográfica Sony Cybershort, bem como para realização da contagem do mobiliário existente, e dimensionamento do espaço.

A segunda parte compreendeu o cálculo do tempo de reverberação do ambiente, utilizando como ferramenta planilha do Excel configurada para realizar o cálculo a partir da equação de Sabine, nesta etapa foi consultado o índice de absorção dos materiais na NBR 12179 (ABNT, 1992) e calculado o tempo ótimo de reverberação com base no método de De Marco (1982).

$\mathrm{Na}$ terceira parte da pesquisa, foram realizadas avaliações comparativas entre o tempo reverberação no local e o tempo ótimo de reverberação obtido, e a partir dessas, foram propostas simulações de novos materiais no ambiente para ser obtida a correção da acústica do auditório, chegando à obtenção do tempo de reverberação ideal no caso da sua função principal que é a palavra falada.

\section{RESULTADOS}

Como o ambiente em questão destina-se à palavra falada, é exigido um tempo de reverberação menor que o de ambiente para grandes concertos ou peças teatrais. Com o auxílio do ábaco (figura 4) a seguir, presente na metodologia de De Marco (1982), obtém-se o Tempo Ótimo de Reverberação (TOR) em função do volume do ambiente estudado, cujo valor é de 0,72 s.

Figura 4 - Ábaco utilizado para o cálculo do TOR.

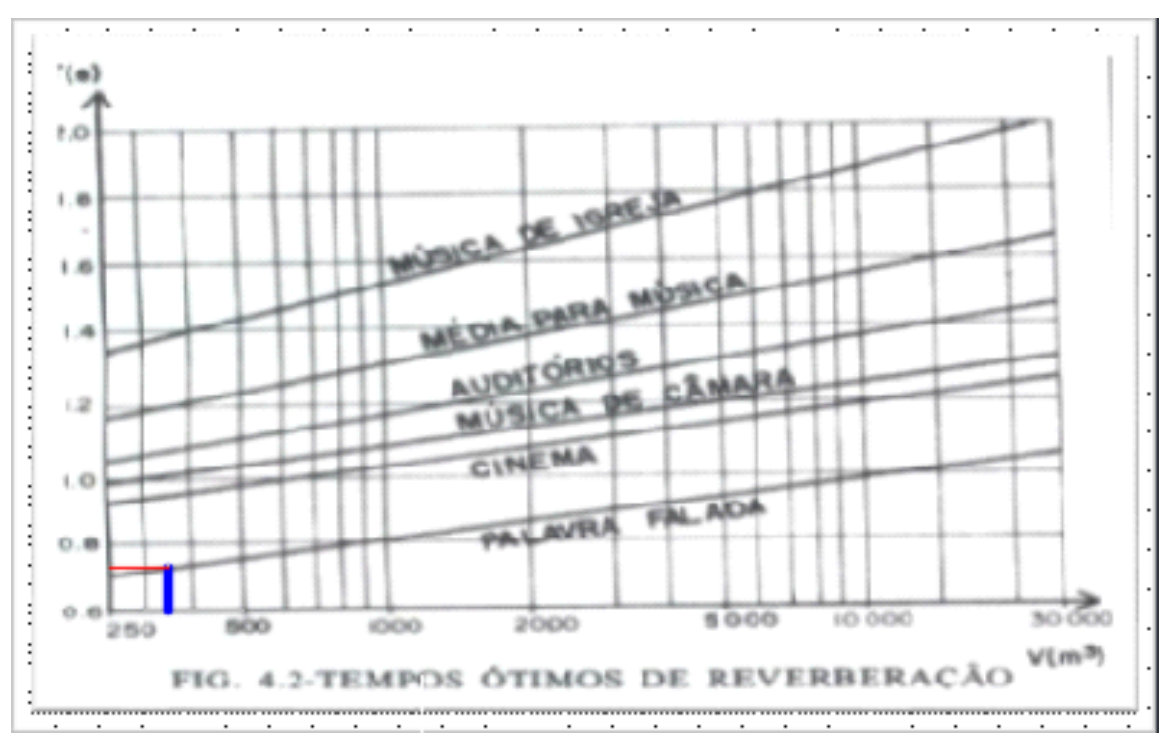

Fonte: De Marco (1982) 


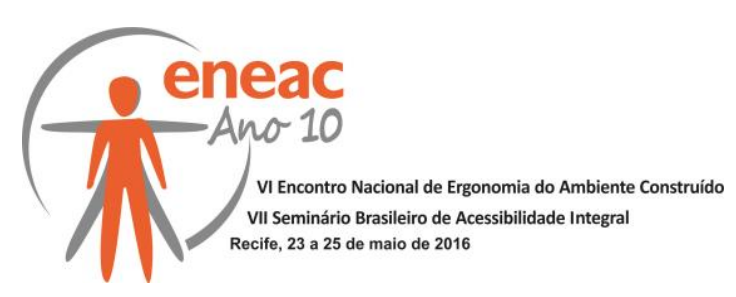

Depois de encontrado o TOR tomando como base a metodologia de De Marco (1982) e após serem obtidos, os valores dos coeficientes de cada material presente no auditório, na NBR 12179 (ABNT, 1992) e em bibliografias especializadas, calculamos o tempo de reverberação da sala para as três frequências da fala, fazendo uso de uma planilha no Excel formatada com a equação de Sabine. Os resultados são mostrados na tabela 5 a seguir.

Tabela 5 - Cálculo do Tempo de Reverberação do recinto.

\begin{tabular}{|c|c|c|c|c|c|c|c|c|c|c|}
\hline \multicolumn{11}{|c|}{ PLANILHA GERAL DE CÁLCULO DE TEMPO DE REVERBERAÇÃO } \\
\hline \multirow{3}{*}{\multicolumn{2}{|c|}{$\begin{array}{l}\text { Amb.: } \\
\text { o Bloco de Direito } \\
\text { das FIP }\end{array}$}} & Larg. & Comp. & Alt. & \multicolumn{2}{|c|}{$\begin{array}{c}\text { Volum } \\
\text { e }\end{array}$} & Ocup. & \multicolumn{2}{|c|}{ V/Ocup } & Tor \\
\hline & & 7,53 & 14 & 3 & \multicolumn{2}{|c|}{323,94} & 98 & \multicolumn{2}{|c|}{3,31} & 0,72 \\
\hline & & \multicolumn{9}{|c|}{ FREQÜENCIA } \\
\hline \multirow{2}{*}{\multicolumn{2}{|c|}{ Item- Especificação }} & \multicolumn{3}{|c|}{$500 \mathrm{~Hz}$} & \multicolumn{3}{|c|}{$125 \mathrm{~Hz}$} & \multicolumn{3}{|c|}{$2000 \mathrm{~Hz}$} \\
\hline & & Si & $\alpha \mathbf{i}$ & Si $x \alpha \mathbf{i}$ & $\alpha i$ & \multicolumn{2}{|c|}{ Si $x \alpha \mathbf{i}$} & $\alpha \mathbf{i}$ & \multicolumn{2}{|c|}{ Si $x \alpha \mathbf{i}$} \\
\hline 1 & Piso cerâmico & 77,72 & 0,010 & 0,777 & 0,010 & 0,77 & & 0,020 & & 554 \\
\hline 2 & Piso carpete simples & 29,02 & 0,250 & 7,255 & 0,010 & 0,29 & & 0,400 & & 608 \\
\hline 3 & $\begin{array}{l}\text { Porta de madeira } \\
\text { compensada fechadas }\end{array}$ & 3,99 & 0,060 & 0,239 & 0,140 & 0,55 & & 0,100 & & 399 \\
\hline 4 & $\begin{array}{l}\text { Paredes frontal em } \\
\text { alvenaria pintada }\end{array}$ & 21,01 & 0,02 & 0,420 & 0,010 & 0,21 & & 0,020 & & 420 \\
\hline 5 & $\begin{array}{l}\text { Paredes lateral direita } \\
\text { alvenaria pintada }\end{array}$ & 21,07 & 0,020 & 0,421 & 0,010 & 0,21 & & 0,020 & & 421 \\
\hline 5 & $\begin{array}{l}\text { Paredes lateral direita } \\
\text { com textura }\end{array}$ & 25,88 & 0,060 & 1,553 & 0,040 & 1,03 & & 0,040 & & 035 \\
\hline 5 & $\begin{array}{l}\text { Paredes lateral esquerda } \\
\text { de alvenaria pintada }\end{array}$ & 20,68 & 0,020 & 0,414 & 0,010 & 0,20 & & 0,020 & & 414 \\
\hline 5 & $\begin{array}{l}\text { Paredes lateral esquerda } \\
\text { com textura }\end{array}$ & 25,88 & 0,060 & 1,553 & 0,040 & 1,03 & & 0,040 & & 035 \\
\hline 5 & $\begin{array}{l}\text { Paredes fundo de } \\
\text { alvenaria pintada }\end{array}$ & 15,78 & 0,020 & 0,316 & 0,010 & 0,15 & & 0,020 & & 316 \\
\hline 5 & $\begin{array}{l}\text { Paredes fundo com } \\
\text { textura }\end{array}$ & 9,45 & 0,060 & 0,567 & 0,040 & 0,37 & & 0,040 & & 378 \\
\hline 6 & Madeira envernizada & 3,35 & 0,140 & 0,469 & 0,240 & 0,80 & & 0,130 & & 436 \\
\hline 7 & $\begin{array}{l}\text { Poltrona estofada, vazia, } \\
\text { revestida com tecido }\end{array}$ & 45,00 & 0,280 & 12,600 & 0,280 & 12,60 & 00 & 0,340 & & ,300 \\
\hline 8 & Púlpito de Madeira & 1,49 & 0,140 & 0,209 & 0,600 & 0,89 & & 0,100 & & 149 \\
\hline 9 & Forro de gesso & $\begin{array}{r}105,2 \\
0\end{array}$ & 0,020 & 2,104 & 0,030 & 3,15 & & 0,050 & & 260 \\
\hline
\end{tabular}




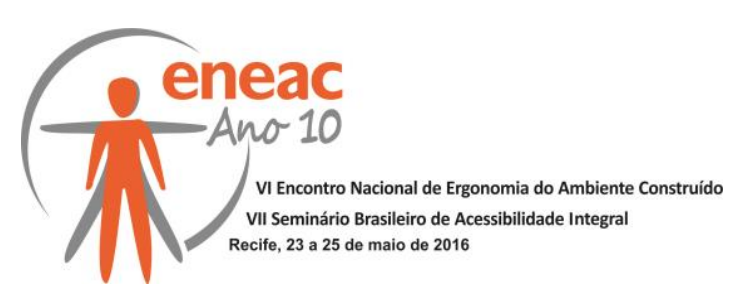

\begin{tabular}{|c|r|r|r|}
\hline $\begin{array}{c}\text { Tempo ótimo de } \\
\text { reverberação (s) }\end{array}$ & 0,72 & 1,06 & 0,72 \\
\hline Absorção total calculada & 28,90 & 22,31 & 38,73 \\
\hline Absorção ideal & 72,44 & 49,28 & 72,44 \\
\hline $\begin{array}{c}\text { Tempo de reverberação } \\
\text { calculado (tr) }\end{array}$ & 1,80 & 2,34 & 1,35 \\
\hline
\end{tabular}

De Marco (1982) recomenda que a diferença entre o tempo de ótimo de reverberação e o tempo de reverberação encontrado não pode ser maior que 0,2s. Pode-se perceber ao analisar os tempos de reverberação que a diferença entre eles é maior que o recomendado em todas a frequências, estando fora dos padrões recomendados, sendo necessária uma correção acústica nas superfícies do auditório, com a utilização de materiais com maior absorção das ondas sonoras.

Para o tratamento acústico foram simuladas correções com a escolha de materiais que realizassem a absorção ideal do som, para se chegar ou aproximar, aos valores dos TOR das frequências de $125 \mathrm{hz}, 500 \mathrm{hz}$ e $200 \mathrm{hz}$. Na primeira simulação optou-se pela aplicação de material absorvente nas paredes laterais, o Eucatex Acústico Ranhurado $19 \mathrm{~mm}$. Os resultados são demonstrados na tabela 6 .

Tabela 6 - Primeira simulação de correção acústica com utilização de Eucatex acústico ranhurado $19 \mathrm{~mm}$ nas paredes laterais.

\begin{tabular}{|c|c|c|c|c|c|c|c|c|c|c|c|c|}
\hline \multicolumn{13}{|c|}{ PLANILHA GERAL DE CÁLCULO DE TEMPO DE REVERBERAÇÃO } \\
\hline \multirow{3}{*}{\multicolumn{2}{|c|}{$\begin{array}{l}\text { Amb.: } \\
\text { Auditório do Bloco de Direito } \\
\text { das FIP }\end{array}$}} & \multirow{2}{*}{$\begin{array}{l}\text { Larg. } \\
7,53\end{array}$} & \multirow{2}{*}{\multicolumn{2}{|c|}{\begin{tabular}{c|} 
Comp. \\
14,34
\end{tabular}}} & \multirow{2}{*}{$\begin{array}{l}\text { Alt. } \\
3\end{array}$} & \multirow{2}{*}{\multicolumn{3}{|c|}{\begin{tabular}{|c|}
$\begin{array}{c}\text { Volum } \\
\text { e }\end{array}$ \\
323,94 \\
\end{tabular}}} & Ocup. & \multicolumn{2}{|c|}{$\begin{array}{c}\text { V/Ocup } \\
\text {. }\end{array}$} & \multirow{2}{*}{$\begin{array}{l}\text { Tor } \\
0,72\end{array}$} \\
\hline & & & & & & & & & 98 & & & \\
\hline & & \multicolumn{11}{|c|}{ FREQÜENCIA } \\
\hline \multirow{2}{*}{\multicolumn{2}{|c|}{ Item- Especificação }} & \multirow[b]{2}{*}{ Si } & \multicolumn{3}{|c|}{$500 \mathrm{~Hz}$} & \multicolumn{4}{|c|}{$125 \mathrm{~Hz}$} & \multicolumn{3}{|c|}{$2000 \mathrm{~Hz}$} \\
\hline & & & $\alpha \mathbf{i}$ & \multicolumn{2}{|c|}{ Si $x \alpha \mathbf{i}$} & \multirow{2}{*}{$\frac{\alpha \mathbf{i}}{0,010}$} & & \multicolumn{2}{|c|}{ Si $x \alpha \mathbf{i}$} & $\alpha \mathbf{i}$ & \multicolumn{2}{|c|}{ Si $x \alpha \mathbf{i}$} \\
\hline 1 & Piso cerâmico & 77,72 & 0,010 & & 777 & & & 0,77 & & 0,020 & & 554 \\
\hline 2 & Piso carpete simples & 29,02 & 0,250 & & 255 & 0,01 & & 0,29 & & 0,400 & & 608 \\
\hline 3 & $\begin{array}{l}\text { Porta de madeira } \\
\text { compensada fechadas }\end{array}$ & 3,99 & 0,060 & & 39 & 0,14 & & 0,55 & & 0,100 & & 399 \\
\hline 4 & $\begin{array}{l}\text { Paredes frontal em } \\
\text { alvenaria pintada }\end{array}$ & 21,01 & 0,02 & & 420 & 0,01 & & 0,21 & & 0,020 & & 420 \\
\hline 5 & $\begin{array}{l}\text { Paredes lateral EUC. } \\
\text { ACUS. RANHURADO }\end{array}$ & 21,07 & 0,500 & & 535 & 0,10 & & 2,10 & & 0,500 & &, 535 \\
\hline 5 & $\begin{array}{l}\text { Paredes lateral EUC. } \\
\text { ACUS. RANHURADO }\end{array}$ & 25,88 & 0,500 & & 940 & 0,10 & & 2,58 & & 0,500 & & .940 \\
\hline
\end{tabular}




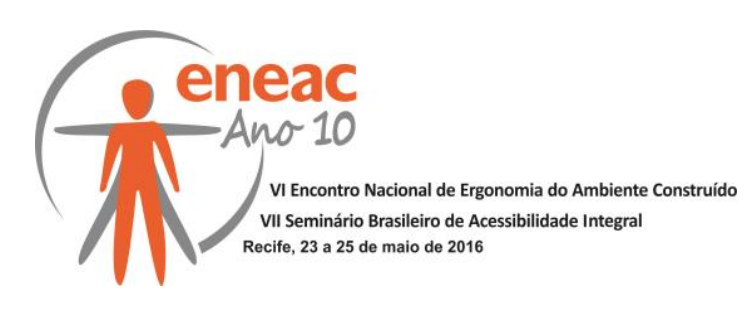

\begin{tabular}{|c|c|c|c|c|c|c|c|c|}
\hline 5 & $\begin{array}{l}\text { Paredes lateral } \\
\text { esquerda EUC. ACUS. }\end{array}$ & 20,68 & 0,500 & 10,340 & 0,100 & 2,068 & 0,500 & 10,340 \\
\hline 5 & $\begin{array}{l}\text { Paredes lateral } \\
\text { esquerda EUC. ACUS. }\end{array}$ & 25,88 & 0,500 & 12,940 & 0,100 & 2,588 & 0,500 & 12,940 \\
\hline 5 & $\begin{array}{l}\text { Paredes fundo de } \\
\text { alvenaria pintada }\end{array}$ & 15,78 & 0,020 & 0,316 & 0,010 & 0,158 & 0,020 & 0,316 \\
\hline 5 & $\begin{array}{l}\text { Paredes fundo com } \\
\text { textura }\end{array}$ & 9,45 & 0,060 & 0,567 & 0,040 & 0,378 & 0,040 & 0,378 \\
\hline 6 & Madeira envernizada & 3,35 & 0,140 & 0,469 & 0,240 & 0,804 & 0,130 & 0,436 \\
\hline 7 & $\begin{array}{l}\text { Poltrona estofada, vazia, } \\
\text { revestida com tecido }\end{array}$ & 45,00 & 0,280 & 12,600 & 0,280 & 12,600 & 0,340 & 15,300 \\
\hline 8 & Púlpito de Madeira & 1,49 & 0,140 & 0,209 & 0,600 & 0,894 & 0,100 & 0,149 \\
\hline 9 & Forro de gesso & 105,2 & 0,020 & 2,104 & 0,030 & 3,156 & 0,050 & 5,260 \\
\hline \multicolumn{2}{|r|}{$\begin{array}{l}\text { Tempo ótimo de } \\
\text { reverberação (s) }\end{array}$} & \multicolumn{3}{|r|}{0,72} & \multicolumn{2}{|r|}{1,06} & \multicolumn{2}{|r|}{0,72} \\
\hline \multicolumn{2}{|c|}{ Absorção total calculada } & \multicolumn{3}{|r|}{71,71} & \multicolumn{2}{|r|}{29,18} & \multicolumn{2}{|r|}{82,57} \\
\hline \multicolumn{2}{|r|}{ Absorção ideal } & \multicolumn{3}{|r|}{72,44} & \multicolumn{2}{|r|}{49,28} & \multicolumn{2}{|r|}{72,44} \\
\hline \multicolumn{2}{|c|}{$\begin{array}{c}\text { Tempo de reverberação } \\
\text { calculado (tr) }\end{array}$} & \multicolumn{3}{|r|}{0,73} & \multicolumn{2}{|r|}{1,79} & \multicolumn{2}{|r|}{0,63} \\
\hline
\end{tabular}

Da comparação entres os resultados das tabelas 05 e 06, é possível verificar que foi obtido um resultado ótimo para a frequência de $500 \mathrm{hz}$ (média) cuja absorção de som chega $71.71 \%$, com porcentual de $1.01 \%$ de diferença entre o tempo de reverberação calculado e o tempo ótimo de reverberação. Porém o material não ofereceu absorção adequada, para das frequências de $125 \mathrm{hz}$ e $2000 \mathrm{hz}$, oferecendo um percentual de absorção de $29.18 \%$ para $125 \mathrm{hz}$, uma vez que sua absorção ideal é de $49.28 \%$. Para a frequência de $2000 \mathrm{hz}$ a absorção garantida pelo material foi de $82,57 \%$, e sua absorção ideal é de $72.44 \%$.

$\mathrm{Na}$ segunda simulação, foi realizada a aplicação Reboco Vermiculite Acústico $3 \mathrm{~cm}$ na parede lateral esquerda e na parede de fundo, bem como inserção de piso de madeira compensada de $2,5 \mathrm{~mm}$ feltro mi $50 \mathrm{~mm} 40 \mathrm{~kg} / \mathrm{m}^{3}$ no perímetro do piso que compreende a arquibancada e o palco. Esses materiais garantem uma absorção de 70,49\% na frequência de $500 \mathrm{hz}, 52,59 \%$ para $125 \mathrm{hz}$ e $71,04 \%$ para $2000 \mathrm{hz}$. Os resultados obtidos estão na tabela 7.

Tabela 7 - Segunda simulação de correção acústica com utilização de madeira no piso e reboco vermiculite.

\section{PLANILHA GERAL DE CÁLCULO DE TEMPO DE REVERBERAÇÃo}

\begin{tabular}{|c|c|c|c|c|c|c|c|}
\hline \multirow{2}{*}{$\begin{array}{c}\text { Amb.: } \\
\text { Auditório do Bloco de Direito } \\
\begin{array}{c}\text { das FIP } \\
$\cline { 2 - 8 }\end{array}\end{array}} & Larg. & Comp. & Alt. & $\begin{array}{c}\text { Volum } \\
\text { e }\end{array}$ & Ocup. & $\begin{array}{c}\text { V/Ocup } \\
\text { - }\end{array}$ & Tor \\
\cline { 2 - 8 } & 7,53 & 14,34 & 3 & 323,94 & 98 & 3,31 & 0,72 \\
\hline
\end{tabular}




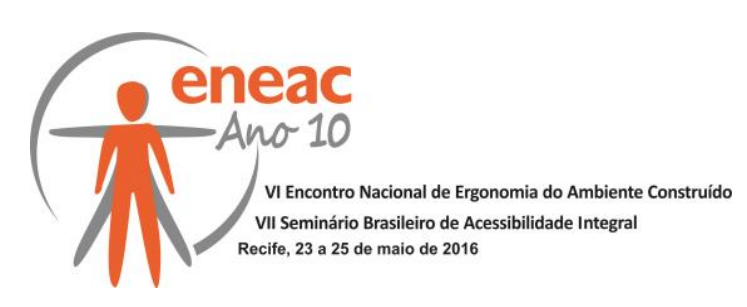

\begin{tabular}{|c|c|c|c|c|c|c|c|c|}
\hline & & \multicolumn{7}{|c|}{ FREQÜENCIA } \\
\hline \multirow{2}{*}{\multicolumn{2}{|c|}{ Item- Especificação }} & \multirow{3}{*}{$\begin{array}{c}\mathbf{S i} \\
77,72\end{array}$} & \multicolumn{2}{|c|}{$500 \mathrm{~Hz}$} & \multicolumn{2}{|c|}{$125 \mathrm{~Hz}$} & \multicolumn{2}{|c|}{$2000 \mathrm{~Hz}$} \\
\hline & & & \multirow{2}{*}{$\begin{array}{c}\alpha \mathbf{i} \\
0,240\end{array}$} & \multirow{2}{*}{$\begin{array}{l}\text { Si } x \boldsymbol{\alpha} \mathbf{i} \\
18,653\end{array}$} & \multirow{2}{*}{$\begin{array}{c}\alpha \mathbf{i} \\
0,210\end{array}$} & \multirow{2}{*}{$\begin{array}{l}\text { Si } x \boldsymbol{\alpha i} \\
16,321\end{array}$} & \multirow{2}{*}{$\begin{array}{c}\alpha \mathbf{i} \\
0,020\end{array}$} & \multirow{2}{*}{$\begin{array}{r}\text { Si } x \alpha \\
1,554\end{array}$} \\
\hline 1 & $\begin{array}{l}\text { Piso MADEIRA COMP. } \\
2,5 \mathrm{~mm} \text { FELTRO MI } 50\end{array}$ & & & & & & & \\
\hline 2 & Piso carpete simples & 29,02 & 0,250 & 7,255 & 0,010 & 0,290 & 0,400 & 11,608 \\
\hline 3 & $\begin{array}{l}\text { Porta de madeira } \\
\text { compensada fechadas }\end{array}$ & 3,99 & 0,060 & 0,239 & 0,140 & 0,559 & 0,100 & 0,399 \\
\hline 4 & $\begin{array}{l}\text { Paredes frontal em } \\
\text { alvenaria pintada }\end{array}$ & 21,01 & 0,02 & 0,420 & 0,010 & 0,210 & 0,020 & 0,420 \\
\hline 5 & $\begin{array}{l}\text { Paredes lateral direita } \\
\text { alvenaria pintada }\end{array}$ & 21,07 & 0,020 & 0,421 & 0,010 & 0,211 & 0,020 & 0,421 \\
\hline 5 & $\begin{array}{l}\text { Paredes lateral direita } \\
\text { com textura }\end{array}$ & 25,88 & 0,060 & 1,553 & 0,040 & 1,035 & 0,040 & 1,035 \\
\hline 5 & $\begin{array}{l}\text { Paredes lateral esquerda } \\
\text { de REB. VERMICULITE }\end{array}$ & 20,68 & 0,370 & 7,652 & 0,230 & 4,756 & 0,480 & 9,926 \\
\hline 5 & $\begin{array}{l}\text { Paredes lateral esquerda } \\
\text { com REB. VERMICULITE }\end{array}$ & 25,88 & 0,370 & 9,576 & 0,230 & 5,952 & 0,480 & 12,422 \\
\hline 5 & $\begin{array}{l}\text { Paredes fundo de REB. } \\
\text { VERMICULITE ACUS. }\end{array}$ & 15,78 & 0,370 & 5,839 & 0,230 & 3,629 & 0,480 & 7,574 \\
\hline 5 & $\begin{array}{l}\text { Paredes fundo com REB. } \\
\text { VERMICULITE ACUS. }\end{array}$ & 9,45 & 0,370 & 3,497 & 0,230 & 2,174 & 0,480 & 4,536 \\
\hline 6 & Madeira envernizada & 3,35 & 0,140 & 0,469 & 0,240 & 0,804 & 0,130 & 0,436 \\
\hline 7 & $\begin{array}{l}\text { Poltrona estofada, vazia, } \\
\text { revestida com tecido }\end{array}$ & 45,00 & 0,280 & 12,600 & 0,280 & 12,600 & 0,340 & 15,300 \\
\hline 8 & Púlpito de Madeira & 1,49 & 0,140 & 0,209 & 0,600 & 0,894 & 0,100 & 0,149 \\
\hline 9 & Forro de gesso & 105,2 & 0,020 & 2,104 & 0,030 & 3,156 & 0,050 & 5,260 \\
\hline & $\begin{array}{l}\text { Tempo ótimo de } \\
\text { reverberação (s) }\end{array}$ & \multicolumn{3}{|c|}{0,72} & \multicolumn{2}{|c|}{1,06} & \multicolumn{2}{|c|}{0,72} \\
\hline \multicolumn{2}{|c|}{ Absorção total calculada } & \multicolumn{3}{|c|}{70,49} & \multicolumn{2}{|c|}{52,59} & \multicolumn{2}{|c|}{71,04} \\
\hline & Absorção ideal & \multicolumn{3}{|c|}{72,44} & \multicolumn{2}{|c|}{49,28} & \multicolumn{2}{|c|}{72,44} \\
\hline \multicolumn{2}{|c|}{$\begin{array}{c}\text { Tempo de reverberação } \\
\text { calculado (tr) }\end{array}$} & \multicolumn{3}{|c|}{0,74} & \multicolumn{2}{|c|}{0,99} & \multicolumn{2}{|c|}{0,73} \\
\hline
\end{tabular}

Pode-se perceber a correção acústica para as frequências de $500 \mathrm{hz}$ e $2000 \mathrm{hz}$ com diferença de 0,2s aproximadamente entre o Tempo de Reverberação Calculado e o Tempo Ótimo de Reverberação após a substituição dos materiais, além de uma acentuada aproximação ao TOR da frequência de $125 \mathrm{hz}$, sendo esta simulação, a que melhor resolve 


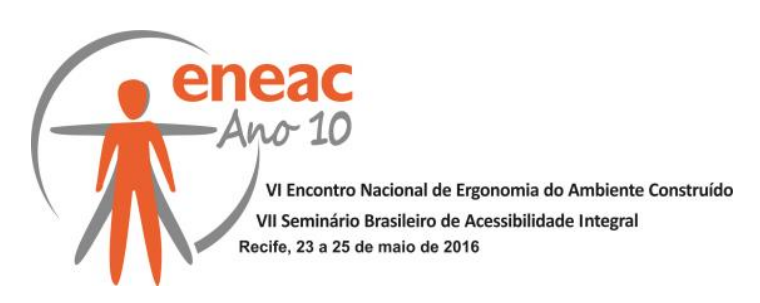

os problemas de propagação do som no recinto, garantindo condições adequadas de audibilidade aos usuários do espaço.

\section{CONCLUSÃO}

Neste trabalho foram analisados os resultados dos cálculos realizados para medição da qualidade acústica de um auditório com capacidade de 98 pessoas e propostas simulações de correções, após ter sido detectado que o mesmo necessita de trato acústico. O potencial de qualidade acústica no objeto de estudo em questão foi evidenciado a partir da comparação do tempo de reverberação calculado com os tempos ótimos de reverberação contemplados em metodologias específicas.

Foi visto que as possibilidades de correção para uma mesma situação são diversas, tendo em vista a grande quantidade de materiais existente com variados índices de absorção. A maioria dos problemas observados em avaliações pós-ocupacionais, que dizem respeito à acústica dos lugares, podem ser evitados ainda mesmo na fase de concepção do projeto com o planejamento e escolha das tecnologias e materiais conforme as necessidades de uso destes espaços.

A realização desta pesquisa, possibilitou a análise dos fatores que influenciam o condicionamento acústico de auditórios que desempenham atividades acadêmicas, além disso foi possível observar a influência das características arquitetônicas nos fenômenos acústicos, gerando uma fonte de pesquisa que garantirá o conhecimento prévio acerca dos elementos que devem ser avaliados e estudados durante o processo de projetação de tais edificações.

\section{REFERÊNCIAS BIBLIOGRÁFICAS}

DE MARCO, C.S. Elementos da acústica arquitetônica. São Paulo: Nobel, 1982

SILVA, Perides. Acústica arquitetônica e condicionamento de ar. 5. ed. Belo Horizonte. EDTAL E. T. Ltda, 2005.

ASSOCIAÇÃO BRASILEIRA DE NORMAS TÉCNICAS. NBR 12179: Tratamento Acústico em Recintos Fechados. Rio de Janeiro, 1992.

SILVA, Liliane. Proposta de correção acústica do auditório central do campus palmas do IFTO. Palmas,2005.

PINTO, Monica. CARACTERIZAÇÃO ACÚSTICA DE GRANDES AUDITÓRIOS: O caso do auditório da FEUP. Dissertação apresentada para obtenção do título de Mestre em Engenharia Civil - 2011/2012 - Departamento de Engenharia Civil, Faculdade de Engenharia da Universidade do Porto, Porto, Portugal, 2012.

BASTOS, Leonardo. Qualidade Acústica de Auditórios. Dissertação apresentada para obtenção do grau de Mestre em Engenharia Civil - Departamento de Engenharia Civil, Universidade de Aveiro, Coimbra, 2010.

R.R.A.E. Regulamento dos Requisitos Acústicos dos Edifícios. 2008. 